\title{
Early Destination Prediction with Spatio-temporal User Behavior Patterns
}

\author{
RYO IMAI, Tokyo Institute of Technology, Japan \\ KOTA TSUBOUCHI, Yahoo Japan Corporation, Japan \\ TATSUYA KONISHI, The University of Tokyo, Japan \\ MASAMICHI SHIMOSAKA, Tokyo Institute of Technology, Japan
}

\begin{abstract}
Predicting user behavior makes it possible to provide personalized services. Destination prediction (e.g. predicting a future location) can be applied to various practical applications. An example of destination prediction is personalized GIS services, which are expected to provide alternate routes to enable users to avoid congested roads. However, the destination prediction problem requires critical trade-offs between timing and accuracy. In this paper, we focus on early destination prediction as the central issue, as early recognition in destination prediction has not been fully explored. As an alternative to the traditional two basic approaches with trajectory tracking that narrow down the candidates with respect to the trip progress, and Next Place Prediction (NPP) that infers the future location of a user from user habits, we propose a new probabilistic model based on both conventional models. The advantage of our model is that it drastically narrows down the destination candidates efficiently at the early stage of a trip, owing to the staying information derived from the NPP approach. In other words, our approach achieves high prediction accuracy by considering both approaches at the same time. To implement our model, we employ SubSynE for state-of-the-art prediction based on trajectory tracking as well as a multi-class logistic regression based on user contexts. Despite the simplicity of our model, the proposed method provides improved performance compared to conventional approaches based on the experimental results using the GPS logs of 1,646 actual users from the commercial services.
\end{abstract}

CCS Concepts: • Information systems $\rightarrow$ Location based services; • Human-centered computing $\rightarrow$ Ubiquitous computing; • Computing methodologies $\rightarrow$ Modeling methodologies; • Networks $\rightarrow$ Location based services;

Additional Key Words and Phrases: Destination prediction, User location, Next place prediction, Sequence modeling, Early recognition

\section{ACM Reference Format:}

Ryo Imai, Kota Tsubouchi, Tatsuya Konishi, and Masamichi Shimosaka. 2017. Early Destination Prediction with Spatiotemporal User Behavior Patterns. Proc. ACM Interact. Mob. Wearable Ubiquitous Technol. 1, 4, Article 142 (December 2017), 19 pages. https://doi.org/10.1145/3161197

\section{INTRODUCTION}

The wide spread use of smartphones has made it easier for service providers to analyze the current context of users, such as current location, weather, and heart rate, and increase the number of applications that provide services according to such context (e.g. context-awareness services). Location data become important data sources for many services because these can be acquired from many devices. Sen et al. [21] investigated the

Authors' addresses: Ryo Imai, Tokyo Institute of Technology, Tokyo, Japan; Kota Tsubouchi, Yahoo Japan Corporation, Tokyo, Japan; Tatsuya Konishi, The University of Tokyo, Tokyo, Japan; Masamichi Shimosaka, Tokyo Institute of Technology, Tokyo, Japan.

Permission to make digital or hard copies of all or part of this work for personal or classroom use is granted without fee provided that copies are not made or distributed for profit or commercial advantage and that copies bear this notice and the full citation on the first page. Copyrights for components of this work owned by others than the author(s) must be honored. Abstracting with credit is permitted. To copy otherwise, or republish, to post on servers or to redistribute to lists, requires prior specific permission and/or a fee. Request permissions from permissions@acm.org.

(c) 2017 Copyright held by the owner/author(s). Publication rights licensed to Association for Computing Machinery. 2474-9567/2017/12-ART142 \$15.00

https://doi.org/10.1145/3161197

Proceedings of the ACM on Interactive, Mobile, Wearable and Ubiquitous Technologies, Vol. 1, No. 4, Article 142. Publication date: December 2017 
recommendation of music according to the current context. Woerndl et al. [23] developed a recommendation system based on collaborative filtering that incorporates the current context, and evaluated it with a point of interest (POI) recommendation system. Examples of commercial applications include myThings ${ }^{1}$ and IFTTT $^{2}$, which provide services that perform actions, such as sending push notifications and e-mails whenever users enter or leave a location by using GPS data.

By combining user-behavior prediction, it is possible to help users save time by preparing the services to be provided in advance based on their preferences and routines. Parate et al. [19] focused on the waiting time to use a mobile application and investigated the preparation of an application beforehand using an algorithm that predicted the next application to be used and when. Okoshi et al. [17] considered the problem that mobile phones require user effort to choose when and where to receive push notifications, and investigated predicting a good time for users to receive notifications.

Predicting the future location of users (e.g., destinations) is one of the most important topics in user-behavior prediction and has attracted much interest among researchers. By predicting destination, it is possible to recommend a detour without having to wait for a query from the user. For example, when a train is delayed, provide advertisements and search words according to the destination, and suggest news articles to read or videos to watch on the way to the destination. For example, T-connect provides agent services ${ }^{3}$ that send traffic information, weather at the destination, etc., without being given the destination, but predicting it based on the travel history, day of the week, and time of day once the car engine starts. Google Now ${ }^{4}$ provides information relevant to a user based on location history, checkout history, and schedule.

In the destination prediction problem, there is a trade-off between prediction accuracy and timing. In particular, there is a trade-off between the percentage of trips completed and the accuracy of destination prediction. In this context, we refer to a trip as the movement from one location to another. For destination prediction in the early stage of a trip, it is necessary to use only information that can always be referred to (e.g., starting location, day of the week, and time of day). However, it is also necessary to predict the destination with high accuracy and therefore, modify the prediction by considering the amount of information that increases as the trip progresses, such as information on the route that has been taken. For this application, it is useless to provide the detour route after the user has encountered a blocked road. Thus, it is necessary to narrow down the destination with high accuracy in the early stage of the trip. Route guidance for a blind man is another example of applying destination prediction. For this application, if early prediction is not possible, it might force the user to take the long wrong way. POI recommendations for tourists on the way to a destination could also be considered. For this application, if destination prediction is delayed, then the opportunity to recommend a POI that the user may be interested in is lost.

In contrast to destination prediction from GPS traces, a variety of work on early recognition in computer vision has been actively explored [20,22]. M. S. Ryoo [20] tackled early recognition systems in human activity recognition from streaming videos because activity recognition is required before fully executing the activity. Early prediction of emotion is also one of the challenging tasks. For example, early prediction of student frustration provides the opportunity to improve the learning environment [15]. However, in destination prediction research there only are a few studies focusing on this trade-off relationship. In this study, we address a new approach to accurately predict destination in the early stage of a trip, which we call early destination prediction with trajectory tracking.

As mentioned above, multiple data sources such as trip trajectories, and user contexts (e.g., day of the week) are used for improving the accuracy of destination prediction [7, 11, 14, 24, 27]. In this study, in order to formulate

\footnotetext{
${ }^{1}$ https://mythings.yahoo.co.jp/

${ }^{2} \mathrm{https}: / / \mathrm{iftt} . \mathrm{com} /$

${ }^{3}$ http://tconnect.jp/detail/3289602/

${ }^{4}$ https://www.google.com/intl/ja/landing/now/
}

Proceedings of the ACM on Interactive, Mobile, Wearable and Ubiquitous Technologies, Vol. 1, No. 4, Article 142. Publication date: December 2017. 
destination prediction as a simple model, it is possible to categorize these sources into two types: trip information obtained from the trip trajectory and user contexts obtained at the starting phase, such as the day of the week and the time of day. Based on how data sources are leveraged; existing destination prediction methods can be classified into two types. One is trajectory-based prediction which is based on trip information, and the other is Next Place Prediction which is based on user contexts.

In the literature, location traces are modeled as trajectories for destination prediction [11, 24, 25]. In this approach, the next position during the trip is inferred from the past trip logs (with Markov assumption [24, 25]), thus the destination candidates are gradually narrowed down as the trip progresses. Hence, the prediction accuracy improves as the trip progresses. For example, the destination cannot be specified at the starting point of the trip (e.g., user home), while the office will be detected as the destination when the user reaches the station closest to one's office. However, these methods have a low accuracy in the early stage of the trip. This stems from fact that these methods predict many potential routes because little information can be used as a reference in the early stage of the trip. For example, if the only information given is that the starting location is at home, many potential routes will be predicted. This constitutes a disadvantage from the viewpoint of early destination prediction.

In addition to destination prediction with trajectory tracking, there has also been much significant work on NPP, which leverages user contexts, such as when and how long the user stayed in a previous location (e.g., the starting location, the day of the week, and time of day) $[7,9,13,16,27]$. By using these methods, predicting the destination with consistent accuracy regardless of the trip progress is possible, because the user contexts at the start of one's trip are fully available. For example, if a user starts a trip at home on Monday morning, one's office can be inferred from this context without difficulty. However, if one's movement gets far from the office but gets close to a hospital, the office should become a less likely destination. Since trajectory information on trips is not employed in the NPP setting, the destination cannot be modified with respect to trip progress. In terms of robust destination prediction from the unexpected trajectories based on user contexts, NPP presents a strong drawback to the trajectory-based approach.

In this study, we focus on developing a new model to solve the trade-off between accuracy and timing. In other words, we consider creating a new model to pursue both the accuracy of prediction at the early stage of a trip and the robustness for unexpected trajectories against user contexts. In this paper, we investigate two conventional approaches: trajectory tracking and NPP, and carefully consider exploiting the advantages of the two approaches. The main contribution of this paper is summarized as follows:

(1) This paper provides a brand-new user destination prediction model based on GPS traced log in users' mobile phones, what we call early destination prediction from trajectory tracking, that improves the performance in terms of the trade-off between accuracy and trip progress. We also demonstrate the limitations of the existing methods.

(2) To the best of our knowledge, our work is the first attempt to factorize complex trajectory tracking with user contextual information for early destination prediction into two simple models, and to formulate the task as the product of the two models in a unified manner. We only need to calculate the result of the destination candidates and their probability. This simplicity provides the capability to choose the options of the models for trajectory tracking and NPP, respectively. In our paper, we employ SubSynE [24] as a state-of-the-art trajectory tracking destination predictor. We also employed a multi-class logistic regression as a state-of-the-art NPP.

(3) We verify the performance of our work using the GPS traces of over $1.6 \mathrm{~K}$ users obtained from commercial services where the trip dataset covers $4,000 \mathrm{~km}^{2}$ around Tokyo. The result demonstrates that our proposed method achieves much better performance in terms of the trade-off between accuracy and trip progress than other state-of-the-art methods, such as SubSynE, and multi-class logistic regression. 
The remaining sections are organized as follows. First, we discuss the problem of destination prediction as well as conventional trajectory tracking for destination prediction and NPP, respectively in Section 2 . To solve the problems with the current prediction methods from the viewpoint of early destination prediction with trajectory tracking, Section 4 presents our model that factorizes the complex early destination prediction task into a combination of the conventional approaches. Section 5 descries the experimental results using GPS traces of $1.6 \mathrm{~K}$ users obtained from commercial services.

\section{Related work}

Though few studies on context aware route based destination prediction have been examined in the literature, Ziebart et al. [4,5] presents a pioneering work for this issue using inverse reinforcement learning. The model presented in $[4,5]$ offers robust destination prediction in a unified manner, however, the complexity of this approach is prone to difficulties in feature design due to the nature of inverse reinforcement learning [12].

In contrast to the complex approach for context aware route prediction, our work relies on two conventional frameworks, the trajectory tracking model and the next place prediction model.

Prediction based on trajectory tracking: Trajectory tracking, which predicts the future trajectory from historical user trajectory, is frequently used for destination prediction for intelligent car navigation tasks. This approach makes it possible to modify the prediction as a trip progresses. Basically, this approach is based on the Markov models of a user trip from one location to the other location [24, 25], Krumm and Horvitz [11] improve the Markov-based model by focusing on user land types and the route efficiency. Since this approach is carefully designed with route prediction rather than destination prediction, it has difficulty in obtaining accurate destination prediction at the early stage of a trip due to the limited trajectory Information. Our work compensates for the disadvantages of this approach by employing user contexts in addition to trajectories.

Next Place Prediction (NPP): NPP has been recently explored in the ubiquitous computing community thanks to the availability of mobile phone location logs. This model extracts user location habits by employing user contexts such as the period of time and day of the week, and then predicts one's location from the leveraged user contexts. Thanks to use of user contexts, the prediction accuracy achieves reliable performance at the early stage of a trip. In the literature, user contexts such as the day of the week, and time of day are frequently used for an accurate prediction from past trip patterns $[13,16]$. In addition to the user contexts mentioned above, the current user current and thelength of stay at the current location are used for improving the accuracy [7, 27]. Logistic regression and decision trees are often used for this task.

To solve the low prediction performance due to the shortage of the trip patterns from the logs of a single user, McInerney et al. [14] propose a probabilistic model called, LocHDP (hierarchical Dirichlet processes extending to user location modeling), which shares the habits of each user. However, NPP could not compensate for the misclassified prediction result even if the user trajectory was provided incrementally with respect to the progress of a trip.

Our work compensates for the shortcomings of NPP by providing robust destination prediction for unexpected trajectories against user contexts.

\section{PROBLEM SETTING AND PROBLEMS WITH CURRENT METHODS IN THIS SETTING}

Before describing our model, this section presents a problem setting of destination prediction with user trajectories and user contexts.

\subsection{Problem setting}

In this paper, destination prediction is defined as the problem of predicting the next destination before reaching the destination during a trip (i.e. from one staying point to another staying point). 
As a preprocessing step, the collection of points of interests (POIs) $\mathcal{L}$ are extracted by discretizing the raw GPS traces using clustering techniques such as DBSCAN methods [6]. Let $L$ be the number of the discretized locations $\mathcal{L}$. The set of destinations $\mathcal{Y}$ are selected from the discretized locations when they have a certain length of staying. Let $K<L$ be the number of destination candidates $\mathcal{Y}$.

In each trip, we assume the sequence of discretized locations can be obtained as $l_{1} \ldots l_{T}$, in which a step is incremented with respect to a change of discretized location, and $T$ is the total number of steps in a single trip.

In this paper, destination prediction is formulated as a classifying set of destination candidates $\mathcal{Y}$ from the trajectory information from $l_{1: t}$ by $t$-th step and the user context $x$ such as the time of day and the day of the week. Specifically, the destination prediction can be formulated as:

$$
\hat{y}=\underset{y}{\arg \max } p\left(y \mid x, l_{1: t}\right),
$$

while early destination prediction pursues accurate prediction at the early stage of each trip $t<<T$.

\subsection{Formulation of current prediction methods and specifying their problems}

Before describing our model, we review the conventional approaches: destination prediction with trajectory tracking, and NPP.

In prediction based on trajectory tracking, the probability of a destination $y$ in step $t \in\{1,2, \cdots, T\}$ is formulated as

$$
p\left(y \mid l_{1: t}\right)
$$

(2) shows that this prediction method takes into account the starting location (that is, location data at $l_{1}$ ) as the user context, but does not take into account the day of the week nor the time of day that cannot be obtained from the location information.

On the other hand, in NPP, the probability of a destination $y$ in step $t \in\{1,2, \cdots, T\}$ is formulated as

$$
p(y \mid x) .
$$

(3) indicates that NPP does not take into account trip information.

As shown here, each approach does not leverage all of the user information. In contrast to the traditional methods, our approach fully exploits all of the information $l_{1: t}$ and $\boldsymbol{x}$ in a unified manner.

\section{PROPOSED METHOD: EARLY DESTINATION PREDICTION WITH SPATIO-TEMPORAL USER-BEHAVIOR PATTERNS}

This section presents our proposed model which pursues early destination prediction. Thanks to the simplicity of our formulation, the model consists of the two approaches mentioned above (that is, destination prediction with trajectory tracking and NPP). We also describe the models leveraged in our proposed model for destination prediction with trajectory tracking and NPP.

\subsection{Factorizing destination prediction with trajectories and user contexts into two approaches}

Owing to the fact that the exploitation of both user contexts and trajectories is required to improve the accuracy of the destination prediction at an early stage of a trip, this section presents how to use all the information systematically.

As an example of one of the naive approaches, we merely seek the maximum a posteriori of destination candidates among the models. Specifically, the model can be formulated as:

$$
p\left(y \mid x, l_{1: t}\right)=\max \left(p(y \mid x), p\left(y \mid l_{1: t}\right)\right) .
$$


However, this model just selects trajectories or user contexts based on the probability of each model, that is, this model is not able to consider both the user trajectories and contexts at the same time, hence the problem for early destination prediction remains unsolved.

To resolve this issue, we rethink the model defined in $p\left(y \mid x, l_{1: t}\right)$. By using the relationship between joint and conditional probability, the model can be factorized into two elements as:

$$
\begin{aligned}
p\left(y \mid \boldsymbol{x}, l_{1: t}\right) & =\frac{p\left(y, l_{1: t} \mid \boldsymbol{x}\right)}{p\left(l_{1: t}\right)} \\
& \propto p\left(y, l_{1: t} \mid \boldsymbol{x}\right) \\
& =p(y \mid \boldsymbol{x}) p\left(l_{1: t} \mid y, \boldsymbol{x}\right) .
\end{aligned}
$$

Note that the first term of the right hand side, i.e. $p(y \mid x)$ is equivalent to NPP. As for the second term: $p\left(l_{1: t} \mid y, x\right)$, it is natural to think the route $l_{1: t}$ is mainly governed by the destination $y$, thus the user context $x$ can be ignored for trajectory modeling. By using this assumption, the probability defined in $p\left(y \mid \boldsymbol{x}, \boldsymbol{l}_{1: t}\right)$ can be formulated as:

$$
\begin{aligned}
p\left(y \mid \boldsymbol{x}, l_{1: t}\right) & \propto p\left(y, l_{1: t} \mid \boldsymbol{x}\right) \\
& \propto p(y \mid \boldsymbol{x}) p\left(l_{1: t} \mid y\right) .
\end{aligned}
$$

Thanks to this assumption, the probability can be factorized into two conventional approaches: trajectory based destination prediction, and NPP. Qualitatively, the model produces a reliable result by using the NPP approach when trajectory information is limited at the early stage of a trip while it also produces a reliable result at the last stage of a trip.

To differentiate our model (6) from the naive one defined in (4), let us demonstrate a typical example qualitatively. Consider a user whose candidate destinations for weekday morning are two choices: the office and the hospital, and the route from home to the office and the supermarket are almost the same. In this case, the candidate destinations are narrowed down to two, the office and the hospital, according to the user context at the time of departure. But in the case of the naive approach, even if the trip progresses, the only changes the candidate can make are to the office and supermarket, that is, the destination cannot be narrowed down even if the trip progresses. This is because the naive approach cannot consider the trip trajectory and user context simultaneously. On the other hand, our approach is carefully designed to deal with two types of information simultaneously therefore, the office is extracted as the destination according to the trip trajectory progress and user context.

Since our model is equivalent to the product of the two models, we are free to select between any two models of NPP and trajectory tracking. In our study, we chose SubSynE [24] as a state of the art trajectory tracking model, and multi-class logistic regression [10] as a state of the art NPP model, respectively. Though SubSynE and multi-class logistic regression are not original to our model, the details of these models are described in the preceding sections in order to fully specify our model.

\subsection{Prediction based on trajectory tracking: SubSynE Algorithm}

3.2.1 Overview of SubSynE Algorithm. In the proposed method, we obtained predictions using the Markov model. Let $\mathrm{M}^{\text {Total }}$ be the $L \times L$ matrix representing the transition probability from state to state. In this context, states are discretized locations. Then, $\mathrm{M}^{\text {Total }}$ is expressed using (7) by using $p_{i \rightarrow j}$ to represent the transition 
probability from $i$ to $j$.

$$
\mathrm{M}^{\text {Total }}=\left(\begin{array}{ccccc}
p_{1 \rightarrow 1} & \cdots & p_{1 \rightarrow j} & \cdots & p_{1 \rightarrow L} \\
\vdots & \ddots & & & \vdots \\
p_{i \rightarrow 1} & & p_{i \rightarrow j} & & p_{i \rightarrow L} \\
\vdots & & & \ddots & \vdots \\
p_{L \rightarrow 1} & \cdots & p_{L \rightarrow j} & \cdots & p_{L \rightarrow L}
\end{array}\right) .
$$

We the optimize transition matrix $M^{\text {Total }}$ ( (7)) by using the SubSynE algorithm [24]. This algorithm optimizes the transition matrix of the Markov model built from the GPS traces. The SubSynE algorithm reduces the calculation cost of the SubSyn algorithm [25]. The Markov model prediction is based on a past route and presents a datasparseness problem. In the SubSyn algorithm, the transition matrix M between adjacent states is first created from partial trajectories obtained by dividing one complete route. By using $M$, the transition matrix $M^{\text {Total }}$ including all transition probabilities from one state to another state is created. This algorithm does not optimize each complete route but rather partial trajectories, and therefore solves the data-sparseness problem. See [24, 25] for the details of this algorithm.

To employ SubSynE in our model, it is necessary to calculate the likelihood of the route for each destination using the SubSynE algorithm. The likelihood of route $l_{1: t}$ for destination $y$ is expressed using (8) with $\mathrm{M}$ and $\mathrm{M}^{\text {Total }}$ optimized by the training data. In this context, $\mathrm{M}$ is the transition matrix between adjacent states. Note that $\prod_{i=1}^{t-1} \mathrm{M}_{n_{l_{i}} n_{l_{i+1}}}$ is common to all destinations $y$ because $\prod_{i=1}^{t-1} \mathrm{M}_{n_{l_{i}} n_{l_{i+1}}}$ depends on only $l_{1: t}$.

$$
\begin{aligned}
p\left(l_{1: t} \mid y\right) & =\frac{\mathrm{M}_{n_{l_{t}} n_{y}}^{\text {Total }}}{\mathrm{M}_{n_{l_{1}} n_{y}}^{\text {Total }}} \prod_{i=1}^{t-1} \mathrm{M}_{n_{l_{i}} n_{l_{i+1}}} \\
& =\frac{p_{n_{l_{t}} \rightarrow n_{y}}}{p_{n_{l_{1}} \rightarrow n_{y}}} \prod_{i=1}^{t-1} \mathrm{M}_{n_{l_{i}} n_{l_{i+1}}} \\
& \propto \frac{p_{n_{l_{t}} \rightarrow n_{y}}}{p_{n_{l_{1}} \rightarrow n_{y}}} .
\end{aligned}
$$

\subsection{Prediction by NPP: Multi-class logistic regression using staying information as feature}

3.3.1 Overview of multi-class logistic regression with user contexts. As the NPP method is utilized in our model, we employ multi-class logistic regression using user contexts such as the day of the week, time of day, and starting location extracted from the use log [10]. In this case, the prediction method is formulated as: (9). In this equation, $f^{(1)}$ is a feature indicating the day of the week by one-hot encoding, $f^{(2)}$ is a feature indicating the time of day by one-hot encoding, $\boldsymbol{f}^{(3)}$ is a feature indicating the starting point label, and $\boldsymbol{w}_{d}=\left(\boldsymbol{w}_{1} \otimes \boldsymbol{w}_{2} \otimes \boldsymbol{w}_{3}\right)^{\mathrm{T}}$ is a weight parameter for the destination $y$.

$$
\begin{array}{r}
f(\boldsymbol{x})=\boldsymbol{f}(\boldsymbol{x})^{(1)} \otimes \boldsymbol{f}(\boldsymbol{x})^{(2)} \otimes \boldsymbol{f}(\boldsymbol{x})^{(3)}, \\
p(y \mid \boldsymbol{x})=\frac{\exp \left(\boldsymbol{w}_{y}^{\top} \boldsymbol{f}(\boldsymbol{x})\right)}{\sum_{d^{\prime}=1}^{K} \exp \left(\boldsymbol{w}_{d^{\prime}}^{\top} \boldsymbol{f}(\boldsymbol{x})\right)} .
\end{array}
$$

Specifically, we designed and used these features as follows:

- Feature representing day of the week

This feature was defined as a one-hot coding in which Sunday is the first dimension, Monday is the second dimension, $\cdots$, and Saturday is the seventh dimension. For example, in the case where the user context $\boldsymbol{x}$ 
provides Monday as the day of the week, this feature for $\boldsymbol{x}$ is expressed as (10).

$$
f^{(1)}(x)=[0,1,0,0,0,0,0] .
$$

- Feature representing the time of day

We divided the day into eight time zones and define this feature as a vector representation, where 0:00 to 3:00 is the first dimension, 3:00 to 6:00 is the second dimension, $\cdots$, and 21:00 to 24:00 is the eighth dimension. In addition, to consider the deviation of time zones, this feature is expressed by Gaussian smoothing to identify which time zone the starting time is close to. For example, in the case where $x$ provides 8:00 as the time of the day, because this time is included the third time zone; this feature for $\boldsymbol{x}$ is expressed as (11).

$$
\begin{aligned}
f^{(2)}(\boldsymbol{x}) & =\left[f^{(2)(1)}, f^{(2)(2)}, \cdots, f^{(2)(8)}\right], \\
f^{(2)(i)}(\boldsymbol{x}) & =\frac{\exp \left\{-\frac{(3-\min (i, 8-i))^{2}}{2}\right\}}{\sqrt{2 \pi}} .
\end{aligned}
$$

- Feature representing the starting location

If a user has $n_{u}$ starting locations after discretizing location data (Section 2), and each starting location is labeled 1 to $n_{u}$, we define the starting location representing the future as a one-hot encoding. For example, in the case where the number of starting locations is 5 and $\boldsymbol{x}$ provides the label of the starting location as 2 , this feature for $\boldsymbol{x}$ is expressed as (12).

$$
f^{(3)}(x)=[0,1,0,0,0] .
$$

By using the features above, $f^{(1)}, f^{(2)}, f^{(3)}$, the probability of a destination $y$ is obtained from (9) To optimize the weight parameter $\boldsymbol{w}_{1}, \cdots, \boldsymbol{w}_{K}$ of (9), we use the L2 norm as a regularization term and the typical gradient methods for machine learning (FOBOS in our implementation [3]).

\section{EXPERIMENTAL RESULTS}

\subsection{Experimental Objective}

In order to determine that the proposed method is more suitable for early destination prediction with trajectory tracking than the current state-of-the-art destination prediction, we compare the performance of our model with the trajectory tracking approach and NPP models, as the proposed method combines these two prediction methods. In addition to evaluating the prediction performance in terms of accuracy, the computational cost of our model is also evaluated from the application perspective.

\subsection{Experimental metric: comparison a prediction accuracy}

To evaluate the performance of the models efficiently, we employ the accuracy versus the ratio of a completed trip as the basic evaluation metric. We represent the percentage of the completed trip as a fraction defined using (13). Let $t$ be the current step and $T$ be the number of total steps. For each trip, we assign $t$ to each fraction, where the value of $t / T$ is closest to the fraction. Note that the step interval with respect to $t$ changes is not constant but 
142:9 depends on the changes of location labels.

$$
\text { fraction }=\left\{\begin{array}{l}
0 \\
0.2 \\
0.4 \\
0.6 \\
0.8
\end{array} .\right.
$$

For example, in the case of $T=4, T=9$, the assignments of $t$ for each fraction are listed in Table. 1. In this

Table 1. Example of assignment of $t$ for each fraction

\begin{tabular}{|l|l|l|}
\hline & $T=4$ & $T=9$ \\
\hline fraction & $t$ & $t$ \\
\hline 0 & 0 & 0 \\
\hline 0.2 & 1 & 2 \\
\hline 0.4 & 2 & 4 \\
\hline 0.6 & 2 & 5 \\
\hline 0.8 & 3 & 7 \\
\hline
\end{tabular}

comparison we demonstrate that the proposed method is suitable for our task by confirming that it can predict the destination with a high accuracy in the early stage of a trip.

4.2.1 Evaluation measures. To specify the performance quantitatively, we employ the two following metrics as the prediction accuracy measures with respect to the fraction.

- Accuracy

This measure reflects the accuracy of the prediction performance obtained from (6) with respect to the fraction. The performance is desired to be highly accurate even when the fraction reaches a small level.

- Percentile of top- $k$ true destinations

From an application perspective, such as online advertisement, and the recommendations related to the destination areas, we also employ top- $k$ true accuracy for the evaluation metric. This metric is inspired by information retrieval. Specifically, this measure clarifies the accuracy of the top- $k$ candidates of a destination. If the ground truth destination is included in the top- $k$ candidates, the model accurately predicts the destination. In contrast to basic accuracy, the top- $k$ metric demonstrates the capacity of each model to narrow down the destination with respect to the fractions.

4.2.2 Comparison methods. In addition to the state-of-the-art destination prediction, we chose four comparison methods as follows.

- Baseline: In contrast to the other models, this method is based on the frequency of predicting the destination regardless of day of the week, or the time of day. No contextual information or trajectory information is leveraged in this model. We referred to it as a baseline in the experiment.

- SubSynE: This model is a part of our model, and serves as a state-of-the-art prediction model based on trajectory tracking. We demonstrate only the likelihood of a path (8). By using this likelihood and the baseline prediction $P(y)$, the probability of a destination predicted by SubSynE is formulated as (14).

$$
p\left(y=d \mid l_{1: t}\right) \quad=\frac{P\left(l_{1: t} \mid y=d\right) P(y=d)}{\sum_{d^{\prime}=1}^{K} P\left(l_{1: t} \mid y=d^{\prime}\right) P\left(y=d^{\prime}\right)} .
$$


- A multi-class logistic regression with user context: This model is a part of our model, and serves as a state-of-the-art NPP model.

- Naive combination: In this model, the destination is obtained from (4), i.e. the destination candidates are specified by using a comparison between the two models evaluated separately. We referred to it as a naive combination in the experiment. This model has the disadvantage that the trip and user contexts, other than trajectories, cannot be considered at the same time.

\subsection{Dataset}

4.3.1 Overview of dataset. We obtained a GPS dataset from an application designed by Yahoo! JAPAN ${ }^{5}$. The GPS data included user ID, latitude, longitude, time stamp (JST), speed, and GPS accuracy. For this study, we leveraged user ID, latitude, longitude, and time stamp.

We collected the GPS data obtained from 1,646 users living around Tokyo from May 1, 2016 to June 30, 2016. For this study, we defined the area around Tokyo as that within the northern latitude 35.5 - 35.9 degrees and east longitude 139.2 - 139.95 degrees (Fig. 1). In this setting, we initially chose 1,690 users who had complete GPS data, and we excluded users who did not have enough trips for training. Specifically, we excluded users whose number of trips was less than five. Finally, 1,646 users were extracted.

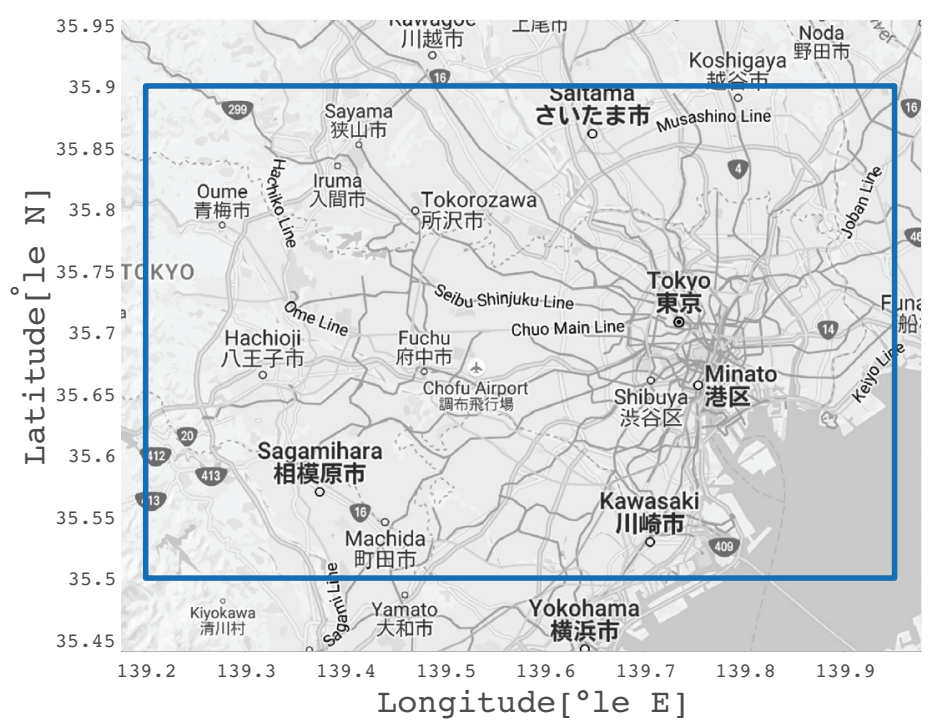

Fig. 1. Defined area of Tokyo

4.3.2 Preprocessing of data. As a preprocessing step, we discretize the raw GPS traces from the dataset by using clustering techniques. Although clustering techniques for GPS traces have been fully explored in the literature, we employ DBSCAN [6] as the clustering technique in our experiment for the following reasons. This clustering does not require the number of clusters in advance, unlike k-means clustering [1]. In addition, DBSCAN is density-based non-hierarchical clustering, the computational quantity can be suppressed $O(n \log n)$

\footnotetext{
${ }^{5}$ https://itunes.apple.com/jp/app/yahoo!-fang-zai-su-bao-zhenya/id481914139
} 
on average when the amount of GPS data is $n$ by combining the data structures suitable for a spatial index such as $\mathrm{R}^{*}$-tree [2]. Owing to the fact that GPS data with few spatially close data can be labeled as noise, the data are suitable for location-based applications, with many examples $[8,18,26]$ (For details on the DBSCAN-clustering algorithm, see [6]). An example of applying DBSCAN clustering to artificial data is shown in Figs. Fig. 2 and Fig. 3. These figures display how to discretize using DBSCAN clustering.

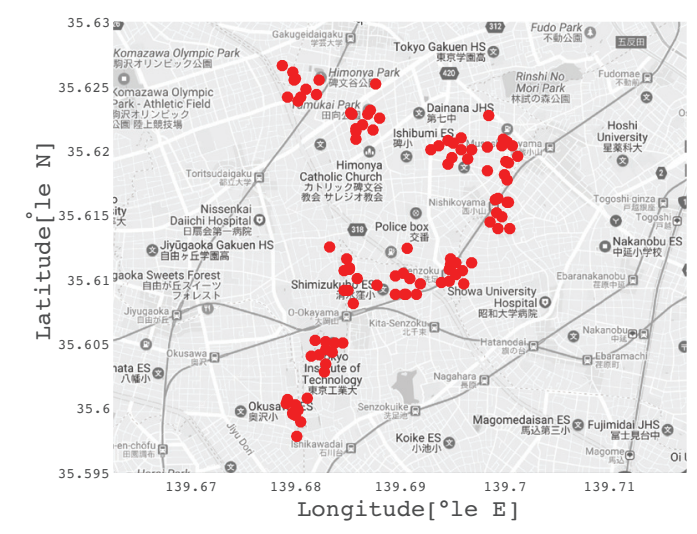

Fig. 2. Artificial data (before clustering)

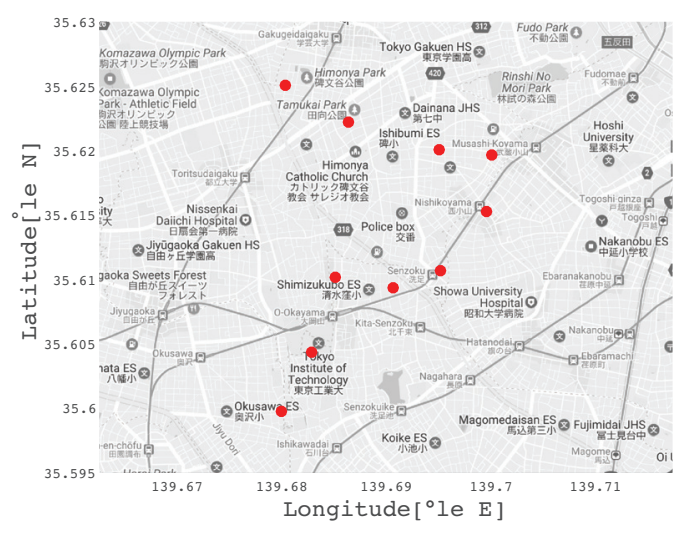

Fig. 3. Artificial data (after clustering)

4.3.3 Extracting candidate of destination and trip. After the clustering phase, a set of destination candidates are selected if users stay at the same location label at least 20 minutes, i.e. $K$ points are selected as the location labels. Note that this preprocessing step from the raw GPS traces to location label generation and destination candidate selection is separately evaluated per user.

\subsection{Experimental result}

We conducted an experiment to compare the accuracy of destination prediction based on the trip percentage completion by using five-fold cross validation for each user. Since the GPS data where the user remained for more than 20 minutes were labelled as "staying". We ignored trips where the starting location and the destination match because the SubSynE algorithm does not work for them. For training data, we conducted grid-based data complementation to solve the problem where GPS data was acquired at unique intervals. Specifically, in a two-dimensional space with the latitude as the vertical axis and longitude as the horizontal axis, we considered grids that passed the line segment between the GPS data at steps $i$ and $i+1$ as those that passed through on this trip and decided that the dataset should include these GPS grids. Fig. 4 shows an example of this data complementation. The number in the upper left corner of a cell is the label number for that cell, the arrows are the direction of trip, the black dots are the GPS data in the dataset, and the white dots are the complemented GPS data.

Fig. 5 shows the statistics of the step length per trip for all users before data completion. The statistical trend of the step length dataset demonstrates that users tend to undertake short distance trips more frequently than travelling long distances.

\subsubsection{Result of prediction accuracy.}



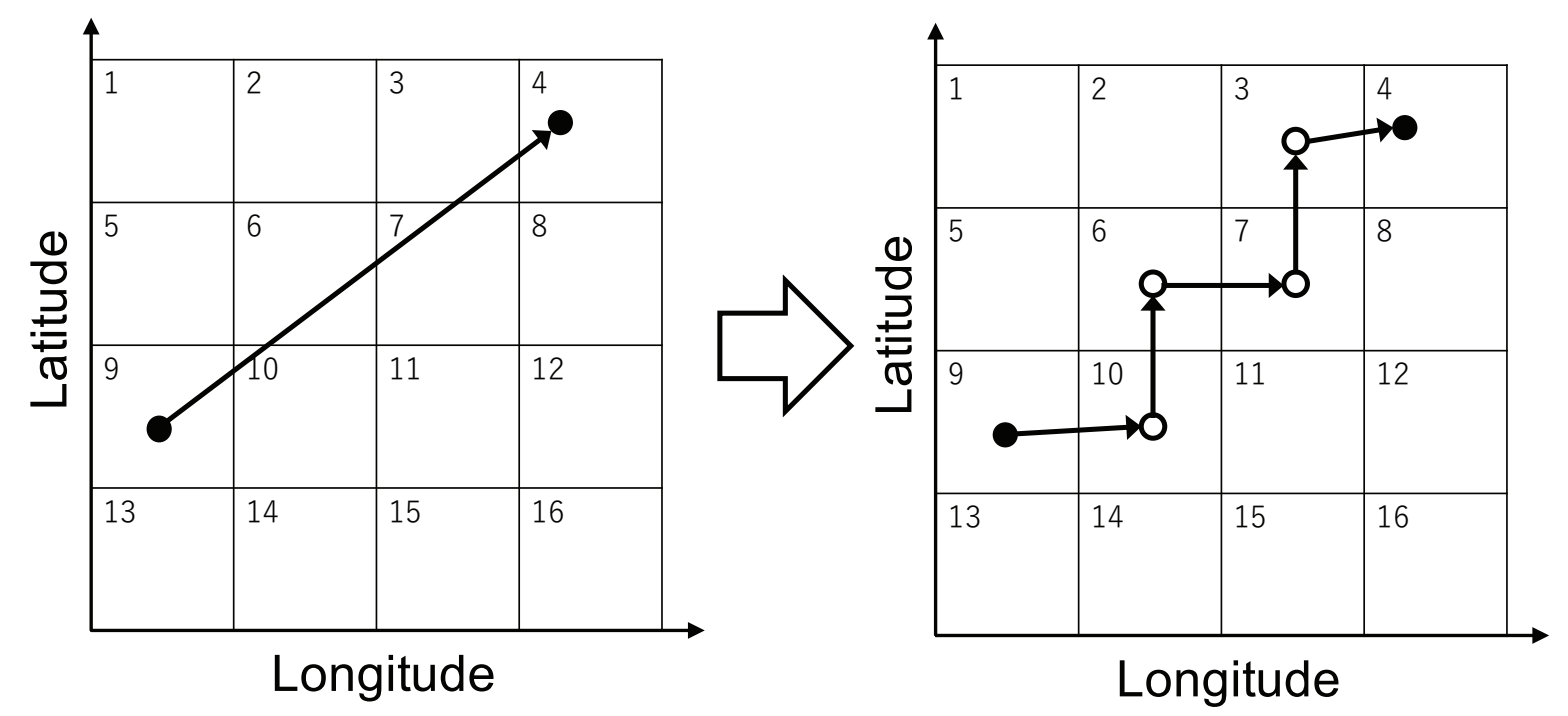

Fig. 4. Example of grid-based data complementation

Accuracy. Fig. ?? shows the experimental results of the accuracy for each percentage of trip completed. The vertical axis is the average of the percentage of the predicted true destination of each user, and the error bars represent the $95 \%$ confidence intervals of this population mean.

First, we confirmed the effect of user trajectories and user contexts on destination prediction via a comparison with the baseline and other methods. With regards to SubSynE specifically, it can be seen that the accuracy is increased as the trip progresses by considering the trip trajectory. On the other hand, focusing on a multi-class logistic regression, the accuracy achieved at the starting phase of trips is relatively high but is constant with respect to trips in progress.

In comparison with SubSynE and a multi-class logistic regression, the proposed method provides better accuracy for any fraction.

Moreover, by comparing the proposed method with the naive combination, it was confirmed that the proposed method achieves better performance than the naive combination, because it considered both user trajectories and the user contexts at the same time. In particular, it can be seen that there is a large difference in the early stage of a trip. In the example of Section 3, even if the trip progresses to a certain degree, the naive combination only changes the destination candidates from the office and hospital to the office and supermarket and cannot narrow down the destination. It can narrow down the office (or supermarket) as a destination at the time the route is changed towards the office or supermarket branches. On the other hand, the proposed method can narrow down the office as the destination at the early stage of a trip by leveraging trajectories and user contexts. This is demonstrated in Fig. 6.

We also investigate why the improvement in accuracy with respect to the trip progress is small in SubSynE, the naive combination, and our model. Fig. ?? shows that there are many trips whose total step length is 4 or less. When the total step is small, SubSynE is not able to integrate all the possible paths, hence the performance of trajectory based models is not incrementally improved, as expected. Hence, many small-step trips may affect the prediction results. In addition, the comparison of the prediction accuracy for small-step trips was not suitable for 


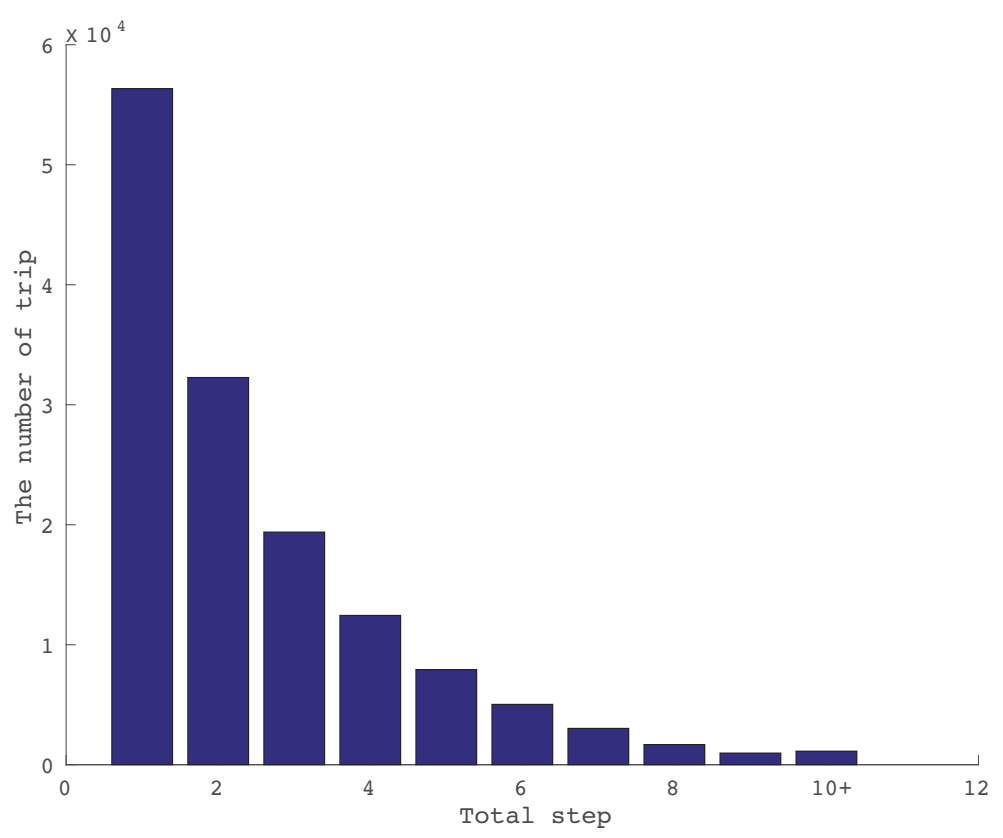

Fig. 5. The number of trips for each total step in the dataset

the experimental objective, that is, to confirm that the proposed method was suitable for our task. Therefore, we extracted trips with total steps of five or more and plotted the prediction results for these trips.

Fig. 7 shows the prediction result for the trips whose total steps are five or more. For SubSynE, the naive combination, and the proposed method, the increase in accuracy during the trip progress was sharp compared with the results shown in Fig. 6. This result confirmed that Fig. 6 was affected by many small-step trips and trip information had the largest effect for long trips.

Percentile of top- $k$ true destinations. In the paragraph regarding Accuracy, we mentioned the results of all trips were not suitable for our experimental objective because these were biased by many small-step trips. For this reason, we only plotted the experimental results for trips with total steps of five or more.

Fig. 8, 9, 10, 11, and 12 show the experimental results of the percentile of top- $k$ true destinations predicted for each $k$ value. The vertical axis represents the percentile of top- $k$ true destinations predicted for each user and the error bars signify a $95 \%$ confidence interval of this population mean as well as the accuracy for each percentage of trip completed. For any fraction, the proposed method always included the true destination at a higher percentile than the other methods, even when $k$ was small. These results show that the proposed method has a high capability of narrowing down a destination by selecting the predicted destinations with a high probability when compared to the other methods.

Discussion for applications. We discuss the advantages of the proposed method in real applications. For example, consider the POI recommendation application for a user trip. If this application requires $70 \%$ accuracy, then this application can recommend POI at a fraction of 0.2 with the proposed method, but cannot recommend any POI with the two existing methods. 
142:14 - R. Imai et al.

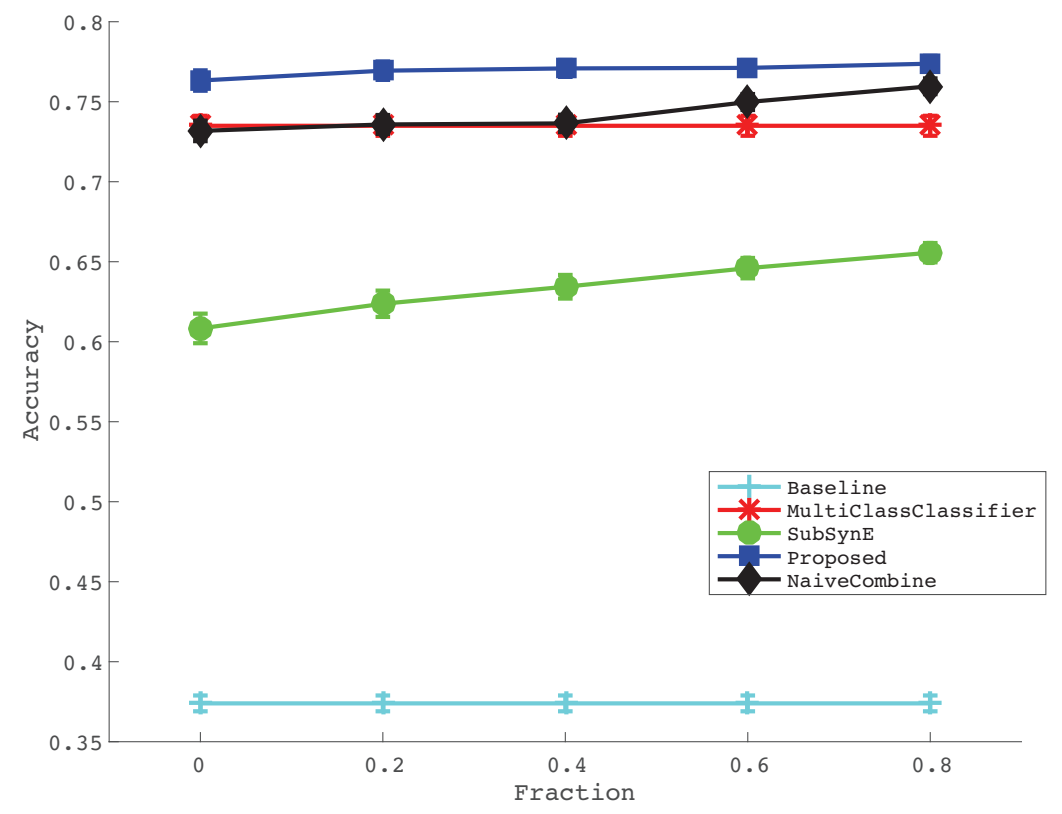

Fig. 6. Accuracy results

As another example, consider displaying web advertisements on a smartphone according to the destination. For simplicity, consider creating one web advertisement for a destination predicted at a fraction of 0.4 , and displaying these on one page. When the probability that the user's true destination will be included in the web advertisement displayed on the page is targeted to $90 \%$ or more, two advertisements are displayed with the proposed method, while four advertisements need to be displayed with the existing methods. Assuming that the advertisement's page occupancy rate is $10 \%$, and one advertisement in the display contains ads that users are impressed with, the percentage of ads that users do not want is only $10 \%$ with the proposed method, and $30 \%$ with existing method. From these results using the above two measures, our model is confirmed to provide robust performance in terms of the early destination prediction task compared with the other models.

\subsection{Measuring computation time}

From the application perspective, we also evaluate the computation time in addition to the accuracy of destination prediction. The computational cost of the training phase and the prediction phase were evaluated and calculated separately. Specifically, we calculate the computational cost of the two phases per user. We employ a single computation node with two CPUs (Intel Xeon E5-2630L v3, $1.80 \mathrm{GHz} \times 2$ ) and $128 \mathrm{~GB}$ of RAM in our environment.

\subsubsection{Result of computation time measurement.}

Training time. The experimental result is shown in Fig. 13. In this figure, the vertical axis depicts the training time [min] and the horizontal axis is trip value of the training set, that is,the number of trips in the training set. Even for users that had the longest training time, the training time was only about $5 \mathrm{~h}$. This result shows that 


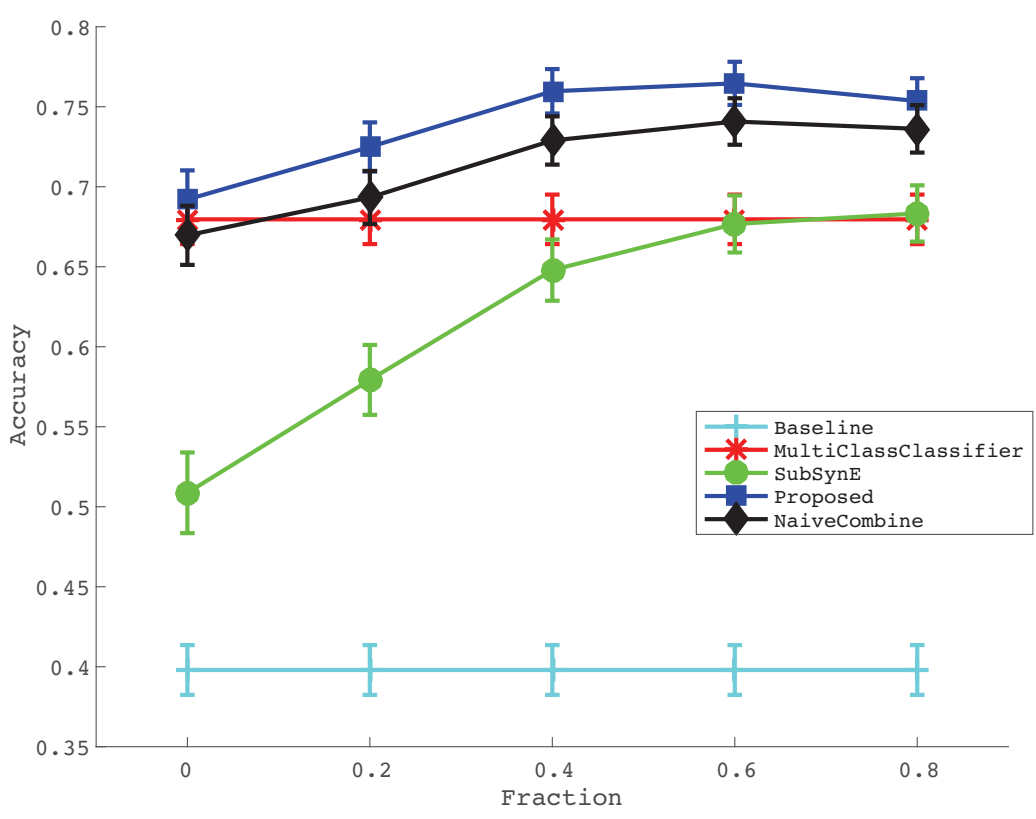

Fig. 7. Accuracy results (total step $\geq 5$ )

the proposed method can predict the destination within a feasible training time. We did not conduct parallel processing in this study, however, it is possible to reduce the training time by leveraging parallel processing.

Prediction time. The experiment result is shown in Table 2. In this figure, prediction time is the average of all predictions. This result demonstrates that the proposed method can generate prediction results immediately based on the current GPS data provided.

Table 2. Prediction time result

\begin{tabular}{|c|c|}
\hline Prediction time $[\mathrm{ms}]$ & 95\% confidence intervals[ms] \\
\hline 3.85 & \pm 0.12 \\
\hline
\end{tabular}

Discussion for applications. Even if the proposed method has a high prediction accuracy, it is meaningless unless the training time and the prediction time are practical. For example, a prediction method that requires 1 year of training to learn from one day of GPS data is not practical. In addition, a prediction method that requires several minutes to generate the results from current GPS data is not suitable for early destination prediction, even if the prediction accuracy is high. Experimental results show that the performance time of the proposed method is sufficient for our task.

Naive combination is inferior to the proposed method in terms of prediction accuracy. It is obvious that the calculation time of naive combination is almost equal to the proposed method. Therefore, there is no reason to use naive combination in practical applications. 


\section{CONCLUSION}

In this paper, a new behavior prediction scheme for early destination prediction with trajectory tracking is presented. Basically, our proposed model fully leverages user context to specify the destination candidates at an early stage of a trip while trajectories are also employed to narrow down the candidates of the destination. To simplify the model, we mathematically factorize this complex task into the two basic models: destination prediction with trajectory tracking and the Next Place Prediction task. In the experiment using $1.6 \mathrm{~K}$ user location logs, we compared our model with the conventional models in terms of prediction performance with respect to the fraction of trips. According to the result, our model achieves better performance than the conventional ones at any stage of the trip. In addition, we measured the computation time of the proposed method and confirmed that this computation time is practical from the application perspective.

The limitation of our work is the preprocessing step when we create a set of destination candidates. Specifically, the current study leverages a type of clustering technique of the raw GPS traces to create a set of candidates. However, the candidates should not be selected from the raw GPS traces of a limited number of users. Destination prediction with an unlimited number of destination candidates should be dealt with as an open problem of this domain.

\section{ACKNOWLEDGEMENT}

The result of this work is obtained from the joint work with Yahoo! JAPAN Corporation; however the discussion in section 2 and 3 was partially supported by JSPS KAKENHI JP25700026, and CREST, JST.

\section{REFERENCES}

[1] K. Alsabti, S. Ranka, and V. Singh. An efficient k-means clustering algorithm. In Proc. of High Prerformance Data Mining 1998 Workshop IPPS/SPDP.

[2] N. Beckmann, H.-P. Kriegel, R. Schneider, and B. Seeger. The R*-tree: An efficient and robust access method for points and rectangles. In Proc. of SIGMOD International Conference on Management of Data 1990, pages 332-331.

[3] J. Duchi and Y. Singer. Efficient online and batch learning using forward backward splitting. F. of Machine Learning Research, pages 2899-2934, 2009.

[4] B. D.Ziebart, A. L.Maas, J. Bagnell, and A. K. Dey. Maximum entropy inverse reinforcement learning. In Proc. of AAAI 2008, pages $1433-1438$

[5] B. D.Ziebart, A. L.Maas, A. K. Dey, and J. Bagnell. Navigate like a cabbie: Probabilistic reasoning from observed context-aware behavior In Proc. of Ubicomp 2008, pages 322-331.

[6] M. Ester, H.-P. Kriegel, J. Sander, and X. Xu. Density-based algorithm for discovering clusters in large spatial databases with noise. In Proc. of KDD 1996, pages 226-231.

[7] H. Gao, J. Tang, and H. Liu. Mobile location prediction in spatio-temporal context. In Proc. of Pervasive Computing 2012 Workshop Nokia Mobile Data Challenge.

[8] S.-S. Ho and S. Ruan. Differential privacy for location pattern mining. In Proc of SPRINGL 2011, pages 17-24.

[9] W. Huang, M. Li, and W. Hu. Hierarchical destination prediction based on GPS history. In Proc. of FSKD 2013, pages 972-977.

[10] T. Konishi, M. Maruyama, K. Tsubouchi, and M. Shimosaka. Cityprophet: city-scale irregularity prediction using transit app logs. In Proceedings of the 2016 ACM International foint Conference on Pervasive and Ubiquitous Computing, pages 752-757. ACM, 2016.

[11] J. Krumm and E. Horvitz. Predestination: Infferring destinations from partial trajectories. In Proc. of UbiComp 2006, pages 243-260.

[12] S. Levine, Z. Popovic, and V. Koltun. Feature construction for inverse reinforcement learning. In Advances in Neural Information Processing Systems, pages 1342-1350, 2010.

[13] C. Manasseh and R. Sengupta. Predicting driver destination using machine learning techniques. In Proc. of ITSC 2013, pages 142-147.

[14] J. McInerney, J. Zheng, A. Rogers, and N. R. Jennings. Modelling heterogeneous location habits in human populations for location prediction under data sparsity. In Proc. of UbiComp 2013, pages 469-478.

[15] S. McQuiggan, S. Lee, and J. Lester. Early prediction of student frustration. F. of ACII 2007, pages 698-709.

[16] A. Nadembega, T. Taleb, and A. Hafid. A destination prediction model based on historical data, contextual knowledge and spatial conceptual maps. In Proc. of ICC 2012, pages 1416-1420.

[17] T. Okoshi, J. Ramos, H. Nozaki, J. Nakazawa, A. K. Dey, and H. Tokuda. Reducing users' perceived mental effort due to interruptive notifications in multi-device mobile environments. In Proc. of UbiComp 2015, pages 475-486.

Proceedings of the ACM on Interactive, Mobile, Wearable and Ubiquitous Technologies, Vol. 1, No. 4, Article 142. Publication date: December 2017. 
[18] G. Pan, G. Qi, and Z. Wu. Land-use classification using taxi GPS traces. In Trans. on Intelligent Transportation Systems, pages 113-123, 2013.

[19] A. Parate, M. Bohmer, D. Chu, D. Ganesan, and B. M. Marlin. Practical prediction and prefetch for faster access to applications on mobile phones. In Proc. of UbiComp 2013, pages 275-284.

[20] M. S. Ryoo. Human activity prediction: Early recognition of ongoing activities from streaming videos. In Proc of ICCV 2011, pages 1036-1043.

[21] A. Sen and M. Larson. From sensors to songs: A learning-free novel music recommendation system using contextual sensor data. In Proc. of RecSys 2015 Workshop LocalRec.

[22] B. U. Töreyin, Y. Dedeoğlu, U. Güdükbay, and A. E. Cetin. Computer vision based method for real-time fire and flame detection. Pattern recognition letters, 27(1):49-58, 2006.

[23] W. Woerndl, C. Schueller, and R. Wojtech. A hybrid recommender system for context-aware recommendation of mobile applications. In Proc. of ICDE 2007, pages 871-878.

[24] A. Y. Xue, J. Qi, X. Xie, R. Zhang, J. Huang, and Y. Li. Solving the data sparsity problem in destination prediction. J. of VLDB 2015, pages $219-243$

[25] A. Y. Xue, R. Zhang, Y. Zheng, X. Xie, J. Huang, and Z. Xu. Destination prediction by sub-trajectory synthesis and privacy protection against such prediction. Proc of ICDE 2013, pages 254-265.

[26] Y.-T. Zheng, Y. Li, Z.-J. Zha, and T.-S. Chua. Mining travel patterns from GPS-tagged photos. In Proc. of MMM 2011 , pages 262-272.

[27] Y. Zhu, Y. Sun, and Y. Wang. Nokia mobile data challenge: Predicting semantic place and next place via mobile data. In Proc. of Pervasive Computing 2012 Workshop Nokia Mobile Data Challenge.

Received May 2017; revised August 2017; accepted October 2017

Proceedings of the ACM on Interactive, Mobile, Wearable and Ubiquitous Technologies, Vol. 1, No. 4, Article 142. Publication date: December 2017. 


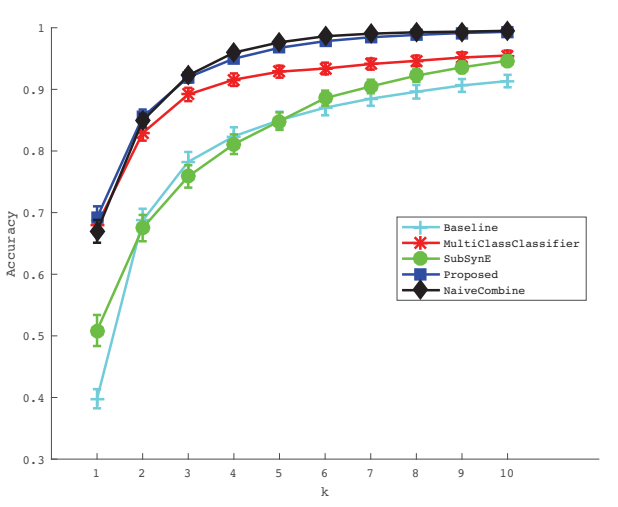

Fig. 8. Percentile results of top- $k$ true destinations predicted for each $k$ value (fraction $=0.0$ )

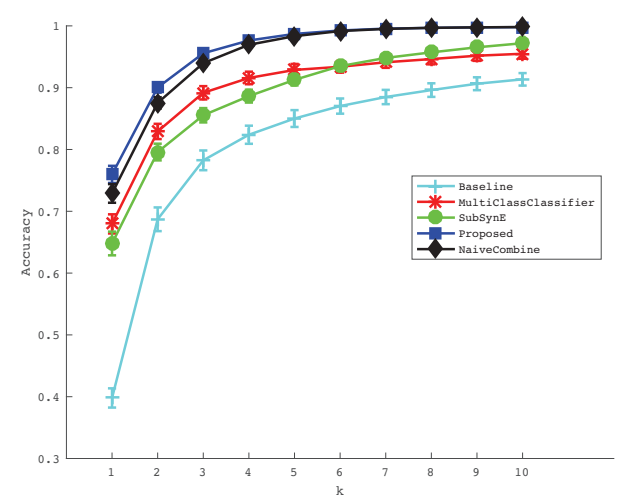

Fig. 10. Percentile results of top- $k$ true destinations predicted for each $k$ value $($ fraction $=0.4)$

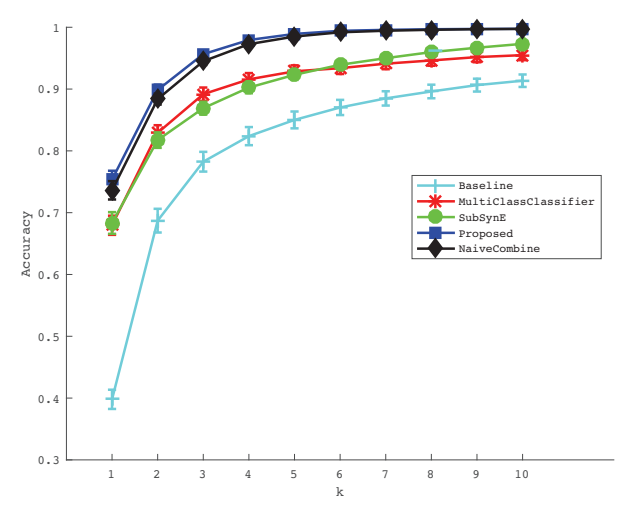

Fig. 12. Percentile results of top- $k$ true destinations predicted for each $k$ value $($ fraction $=0.8)$

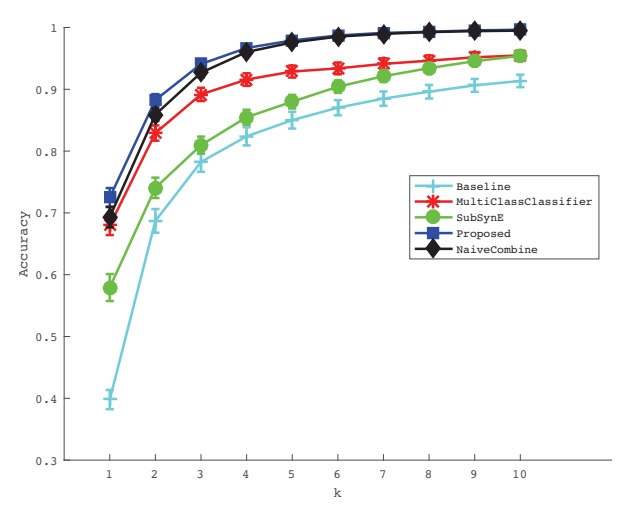

Fig. 9. Percentile results of top- $k$ true destinations predicted for each $k$ value (fraction $=0.2$ )

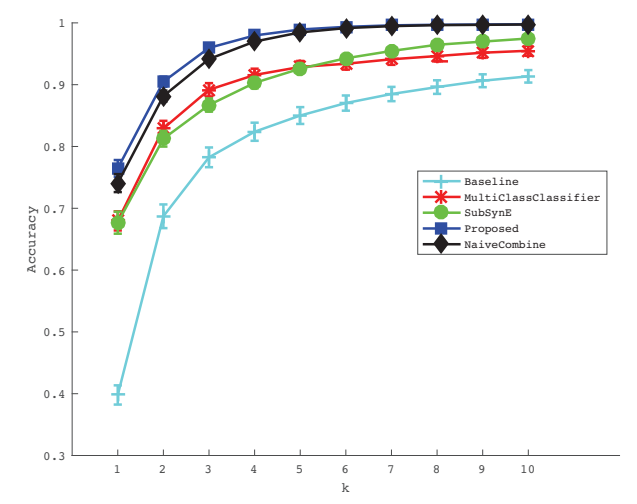

Fig. 11. Percentile results of top- $k$ true destinations predicted for each $k$ value (fraction $=0.6$ )

Proceedings of the ACM on Interactive, Mobile, Wearable and Ubiquitous Technologies, Vol. 1, No. 4, Article 142. Publication date: December 2017. 


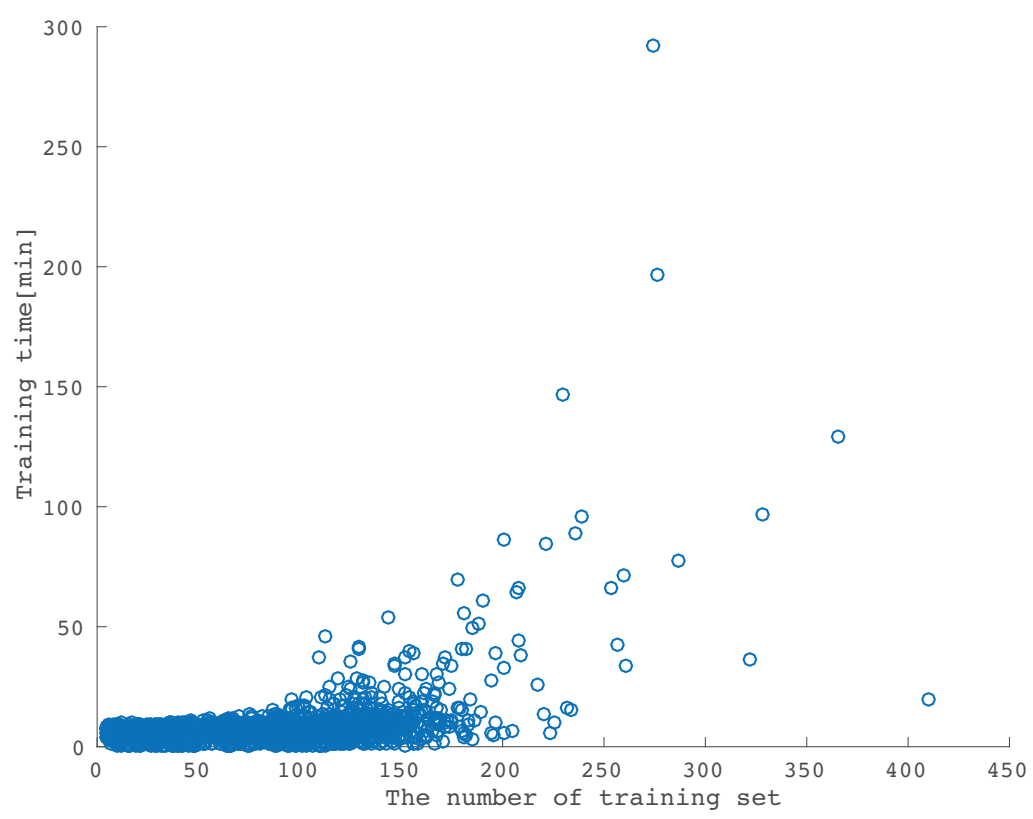

Fig. 13. Training time results by training set trip value 